\title{
El deber moral de los estados y ciudadanos de preservar la sustentabilidad
}

\section{Resumen}

Considerando que formular el deber ser de las cosas - la ética - es una de las tareas de la Filosofía este trabajo busca claves interdisciplinarias para establecer el deber ser ambiental. Para ello analiza algunos problemas que afectan al ambiente como espacio "natural” y humano. ¿Dónde hallar la clave en la economía, la ciencia, la política? Si bien la reflexión tiene un origen ético y busca respuestas políticas, se detendrá sobre todo en el concepto de sustentabilidad.

Palabras-clave: Ética. Política. Ambiente. Desarrollo sostenible. Economía.

\section{Resumo}

O dever moral dos estados e dos cidadãos para preservar a sustentabilidade

Considerando que formular o dever ser das coisas - a ética - é uma tarefa da Filosofia, o presente trabalho busca chaves interdisciplinares para estabelecer o dever ser ambiental. Para tanto, analisa alguns problemas que afetam o meio ambiente como espaço "natural" e humano. Onde encontrar a chave da economia, da ciência, da política? Ainda que a reflexão tenha uma origem ética e busque respostas políticas, se deterá sobretudo no conceito de sustentabilidade.

Palavras-chave: Ética. Política. Meio ambiente. Desenvolvimento sustentável. Economia.

\section{Abstract}

\section{The moral duty of states and citizens to preserve the sustainability}

Taking into consideration that formulating what the nature of things must be - ethics - is a Philosophy task, this work looks for interdisciplinary keys to establish the environmental must-be. To do this, it analyzes some problems which affect the environment as a "natural" and human space. Where can we find the economy, science and politics key? Although the reflection has an ethical origin and seeks political responses, it will address all the concept of sustainability.

Key words: Ethics. Politics. Environment. Sustainable development. Economy.

Doutora maria3729@hotmail.com - Consejo Nacional de Investigaciones Científicas y Técnicas/Universidad de Buenos Aires (UBA), Buenos Aires, Argentina.

\section{Correspondência}

Av. Rivadavia 6.646 $2^{\text {do }}$ piso, 1.406. Ciudad de Buenos Aires/Argentina. 
¿No deberíamos admitir que buena parte de lo que llamamos producción es de hecho extracción?

C.Sauer

Dos mil años les llevó al Pueblo Elegido... retornar a la Tierra prometida.

Le ha tomado sólo 52 años convertir la tierra de leche y miel en un país de ríos con espuma, de aguas carcinogénicas y de peces moribundos

S. Kiley

La filosofía tiene desde sus orígenes dos misiones paralelas: una describir lo que hay, es decir ocuparse con el ser de las cosas, y otra formular el deber ser. No siempre consideramos que lo que es, lo que hay, lo que sucede a nuestro alrededor y en nosotros mismos debe ser de esa manera. Es más, escuchamos casi todo el tiempo que el mundo que nos ha tocado vivir, el ambiente que venimos construyendo estos últimos siglos, no es aquél en que los humanos puedan alcanzar su plenitud. Es por ello que este trabajo desarrollará:

1. algunas cuestiones conflictivas en relación con el ambiente, entendiendo por tal el mundo que nos rodea con todas sus problemáticas y;

2. algunas reflexiones sobre el deber ser, es decir sobre las pautas ético-políticas desde las cuales resolver esos conflictos.

Procuraré evitar todo catastrofismo y pesimismo así como caer en lugares comunes que convierten a la ética en ese tópico al que todos acuden para tranquilizar sus conciencias pero que no obliga a cambiar las conductas. También procuraré evitar la utopía. Aunque ésta no puede estar ausente, me atendré a situaciones y a respuestas ético-políticas concretas. No me detendré tampoco en discusiones en relación a la primacía del hombre sobre la "naturaleza" o viceversa, no sólo porque implicaría otro tipo de desarrollo sino porque además considero que es una discusión inútil cuando lo que está en juego son decisiones políticas que afectan a su vida y a la de su comunidad y que en ello está involucrada necesariamente la "naturaleza". Ésta es constitutiva de la vida del hombre, no algo agregado y ajeno a la misma. El mundo "natural" (espacio que comparten hombres animales, plantas, tierra, espacios en que las distintas especies se desarrollan en relación con su origen común, la tierra) es el hábitat del hombre, es decir su condición de vida fundamental. El hombre no vive solo, por ello no puede prescindir ni de otros hombres ni del mundo del que forma parte constitutiva. Los intereses de la "naturaleza" y los del hombre deben discutirse simultáneamente.

\section{Los responsables}

Adueñándose de los conflictos que afectan al mundo que habitamos, ha proliferado una cantidad de "expertos" que confiamos que sean los que saben y los que habrán de comunicarnos el logos, de la casa que habitamos en común, es decir tendrán la última palabra en ecología ${ }^{1}$. Recurrir a expertos significa recabar soluciones en la ciencia, sin embargo, la primer respuesta a los problemas que surgen de la aplicación de la tecnología y la biotecnología al mundo del que formamos parte, debe ser ética y no científica, es decir debe provenir del compromiso y la responsabilidad de todos los que habitamos este mundo, además de los expertos.

Mae-Wan Ho afirmó en el año 2000, nuestra intención no es que se detenga la investigación, sino que ella deje de hacerse entre cuatro paredes, de espaldas a la sociedad, y sólo en función de los intereses de las grandes corporaciones. Los cientificos deberíamos pasar entre seis y doce meses con organizaciones sociales seleccionadas por nosotros, antes de acometer cualquier proyecto de investigación. Eso nos ayudaría a incluir las necesidades de la sociedad en nuestros objetivos científicos. Los científicos deberíamos mantener una intensa discusión sobre cómo podemos ser útiles a la sociedad, antes que estar tan ocupados especulando acerca de las potencialidades comerciales del resultado de nuestro trabajo ${ }^{1}$.

Las respuestas de los expertos en ecología resultan frecuentemente contradictorias cuando se trata de decidir el límite entre beneficiar a la "naturaleza" y a los humanos. Esto nos lleva a preguntarnos si el más apto para considerar qué hacer no es el afectado por el uso de la tecnología: el habitante de las ciudades cada vez más contaminadas, quien ha perdido todos sus bienes y vive en riesgo permanente por las inundaciones producidas por un mal cuidado de la tierra ${ }^{2,3}$, o quien se ve condenado a la infertilidad o ve a sus hijos enfermos por haber estado en contacto con ciertos fertilizantes o herbicidas como el glifosato ${ }^{4-6}$ y plaguicidas organoclorados ${ }^{7,8}$.

Son los marginados generados por una sociedad organizada sobre bases liberales-capitalistas quienes pueden comprender mejor lo que significa estar obligado a sobrevivir a costa, no sólo del futuro, sino incluso del presente. Si bien es cierto que se puede medir y proyectar el daño al futuro con mayor precisión por medio del conocimiento experto o científico, también lo es que son los ciudadanos comunes, las personas sometidas a la agresión permanente de parte de un mundo cada vez más hostil, en que se sienten cada vez más como extraños, los 
que podrán valorar más acabadamente los problemas y las respuestas posibles.

Somos los ciudadanos y ciudadanas quienes podemos diferenciar entre catástrofes "naturales" y las provocadas por la codicia del hombre, por el uso de armas cada vez más refinadas o "inofensivos experimentos atómicos" por ejemplo o por prácticas que extinguen especies milenarias, o transformaciones biotecnológicas que llevan a la destrucción de la biodiversidad cuyas consecuencias a corto o largo plazo ignoramos a ciencia cierta pero intuimos mirando lo que ocurre a nuestro alrededor. La ciencia puede dar algunos elementos de juicio que, contra la práctica corriente, deben ser considerados a la luz de las problemáticas de los pueblos; y asimismo la ciencia podrá solucionar algunos problemas, muchos de los cuales los ha creado ella misma, pero los fundamentales no caen bajo su incumbencia.

Identificar los problemas y elegir las soluciones como seres humanos afectados, como no expertos, implica en primer lugar la responsabilidad individual y colectiva que nos cabe en su existencia, en su persistencia, en su proyección hacia el futuro y en segundo lugar actuar políticamente, desde la solidaridad, y poniendo al bien común por encima de los intereses particulares y partidarios, con la convicción de que el bien para todos supone necesariamente el bien para cada uno. Esto es precisamente lo que nos pide cada vez más la bioética, no sólo a los bioeticistas sino a cualquier persona: que como sociedad tomemos conciencia de nuestro derecho a un trato igualitario en medio de las diferencias y de nuestra obligación de participar en las distintas instancias de decisión frente a cuestiones que afectan nuestro presente y nuestro futuro. Las decisiones políticas deben sostenerse sobre principios éticos, pero su materialización debe tener en cuenta gradualidades, contextos, condiciones a veces ineludibles, sin embargo ello no debe impedir que el bien común esté por sobre cualquier interés individual.

Se cita en relación a esta problemática a Max Weber quien diferenciaba la ética de la convicción que rige a nivel individual y la de la acción que debe además tomar en cuenta las consecuencias que dependen de los contextos. Esta diferenciación no implica que Weber haya sostenido que actuar estratégicamente implica olvidar los valores o principios éticos ${ }^{9}$. Como parte de una comunidad cada uno debe identificar, denunciar y combatir con la ley y la ética a aquellos que detentan poder en la sociedad, que actúan desde sí mismos y sus intereses, desde principios de acción no solidarios sino individuales, que ignoran voluntariamente su responsabilidad respecto a las necesidades de la gente.

Pero es preciso recordar que en la democracia la política no es sólo cuestión de los poderosos y los gobiernos sino que sobre todo es cuestión de los ciudadanos. Los gobiernos enfrentan sin duda tensiones entre los intereses de corporaciones, empresas, bolsas, y las necesidades de la gente y deberán, con el auxilio y apoyo de los ciudadanos, lograr acuerdos estratégicos sin duda, tal cual lo aconseja Apel ${ }^{10}$. Pero es obligación de los ciudadanos que esos acuerdos sean celebrados bajo la salvaguarda de que no triunfen los intereses particulares sino los derechos comunes. Ante el poder disminuido de los estados nacionales el poder de la ciudadanía debe ser pensado hoy globalmente como posibilidad de una sociedad global. El Internet puede ser un buen medio para lograrlo. Pero es irrenunciable hacerlo sosteniendo algunos principios como el de defensa de la dignidad humana, es decir de respeto por igual de todos y cualquiera de los humanos y el reconocimiento de la naturaleza como portadora de derechos.

El resultado político incuestionable será aquel en que todos los sectores de la población habrán tenido participación, y en el que se haya respetado por sobre todo la igualdad en el beneficio común, incluido el beneficio para la biosfera, bajo el supuesto que lo que es bueno para todos lo es para cada uno. Si priman la dignidad humana y la igualdad, si se reconoce el valor de la naturaleza como tal, se estará defendiendo la verdad por sobre el poder, y la solidaridad por encima del lucro. No es aceptable separar, en el ejercicio del poder, la responsabilidad de la convicción, dejando abierta la puerta para que los estados aduzcan condicionamientos supranacionales, juegos económicos, circunstancias globales, para ignorar las necesidades de los pueblos y la tierra. Es en la coincidencia de la convicción con la responsabilidad donde hallamos la vigencia de la verdad y la justicia, tanto en el plan público como en el privado. Son los expertos, los pueblos, los individuos, pero sobre todo los estados, quienes deben responsabilizarse por mantener un mundo donde todos podamos proyectarnos hacia el futuro.

\section{La economía}

Quien crea que el crecimiento exponencial puede durar eternamente en un mundo finito, es un loco o es un economista. Kenneth Bouldign 
No cabe duda que enfrentamos un problema de equilibrio ecológico, es decir un problema en que la razón debe buscar respuestas para que la casa, el hogar (oikos), sobreviva con todos los que la poblamos. Esto no sólo afecta a América Latina sino a todo el planeta por consiguiente la pregunta es si las responsabilidades políticas y económicas deben ser compartidas por todos por igual. En 1968 Gunnar Myrdal, premio de economía de 1974 dijo: No me cabe duda que en cinco o diez años habrá un movimiento popular en los países ricos que presionará sobre el Congreso y la Administración estatal para que haga muchas cosas para solucionar los problemas del medio ambiente. Pero no sucederá lo mismo en la mayoría, tal vez en ninguno, de los países subdesarrollados ${ }^{11}$.

Cuarenta años después la historia lo desmiente, la economía estándar de lineamientos capitalistas, en los países industrializados, ignora los problemas ambientales en general y en particular, en tanto y en cuanto el mundo "natural" sigue siendo para ellos "recurso", fuente de materia prima o energía. En cuanto a los países no industrializados van perdiendo la relación primaria y de parentesco con la tierra y sus misterios que fue largamente considerada por la antropología académica como una manifestación de primitivismo. Precisamente en su búsqueda de salir de ese primitivismo buscan el "desarrollo" que asocian la industrialización y participación en el mercado global. Su valoración tradicional de la "naturaleza" y la relación del humano con ella, se viene convirtiendo cada vez más en una declamación de tipo romántica o folclórica.

Paralelamente los países que se autodenominan desarrollados consideran que ciertos problemas como el cambio climático pueden resolverse negociando con los países en desarrollo por la emisión de $\mathrm{CO}_{2}$. Los países en desarrollo por su parte responsabilizan a los desarrollados por la emergencia ambiental pero al mismo tiempo no pueden evitar desear y buscar un mayor nivel de vida según los estándares consumistas. Para ellos, entonces, el aumento de emisión de $\mathrm{CO}_{2}$ o la producción de materia prima agroganadera mediante el uso de biotecnología que los países desarrollados evitan por la degradación de los suelos, resultan en índice de desarrollo ${ }^{12,13}$.

La urgencia con que son presentadas las cuestiones económicas y sobre todo las financieras en todo el planeta como catástrofes históricas, son una pantalla que impide comprender que esas catástrofes son juego de niños frente a una naturaleza que ya no puede reaccionar para revertir la situación. El riesgo no pasa por la bolsa de valores de Tokio o Nueva York sino que toda la "naturaleza", con los hombres incluidos, está en riesgo, todos seremos perjudicados y perderemos: no habrá ganadores en este proceso "depredador". El 22 de abril de 2013, Ban Ki Moon, secretario general de la Organización de las Naciones Unidas (ONU) advirtió: Tenemos que afrontar la dura realidad de que nuestro planeta está en peligro...estamos socavando nuestro único hogar y nuestra supervivencia ${ }^{14}$.

El menoscabo de la "casa", de los ecos como habitación ${ }^{15}$, es decir como lugar privilegiado de referencia de la vida humana, puede ser calificado como un "mal absoluto" que está afectando a toda la humanidad, aunque los pobres lo sufran primero. Esto recuerda la expresión kantiana con la que denomina al mal concomitante a la condición humana y que sólo puede ser superado por la gracia divina ${ }^{16}$. Lo que agrava la situación es que más allá de una ecología declamada, en los países del Norte no solamente se ignora la responsabilidad ético-política tanto a nivel gubernamental como a nivel popular, sino que el marco legal privilegiado para solucionar los conflictos de este tipo es comercial:

Todo conflicto respecto a cuestiones ambientales... no posee un espacio especializado donde dirimirse, los únicos que se conocen son los espacios de solución de controversias que prevén las legislaciones comerciales, como el de la Organización Mundial del Comercio (OMC) ${ }^{17}$.

La sustentabilidad resulta planteada y, en este marco, es una cuestión de lucro, la resultante de políticas económicas en que todo se resuelve en el mercado. En el año 1972 Meadows elaboró con un grupo de colaboradores un informe Los límites del crecimiento, que afirmaba que en 100 años el planeta entraría en colapso, dado que en un planeta limitado, las dinámicas de crecimiento exponencial (población y producto per cápita) no son sostenibles ${ }^{18}$. La publicación supuso un decisivo aviso sobre las posibles consecuencias indeseadas del crecimiento económico. Treinta años más tarde, en 2002, replantearon y mejoraron su modelo de investigación en un nuevo estudio ${ }^{19}$.

La posible solución a ese colapso era "crecimiento cero" o "estado estacionario", deteniendo el crecimiento exponencial de la economía y la población, de modo que los recursos naturales que quedan no sean mermados por el crecimiento económico y de esa forma puedan perdurar más en el tiempo. En 2002 sostenía la misma tesis y afirmaba que la Tierra sólo puede mantener a mil millones de personas con el nivel de vida del País Vasco, esto sig- 
nificaba entonces que hacían falta seis planetas para mantener los 6.500 millones de personas vivientes. La solución que proponía y sigue proponiendo es una brusca caída del crecimiento que era y sigue siendo índice de desarrollo. La teoría económica del decrecimiento está en consonancia con esta idea ${ }^{20}$.

De modo que lo que vemos es que cuando el planteo económico tiene que ver con el desarrollo y éste sólo con el aumento del Producto Bruto Interno (PBI): multiplicar ganancias, mantener reservas monetarias, dominio en el mercado. Cuidar el ambiente y a los hombres que lo pueblan no es rentable. Por eso no se trata de enfrentar desarrollo con ética sino ética con economía, o mejor aún con un planteo de la economía que paradójicamente, considerando su etimología, ignora todo lo que tiene que ver con la concepción del mundo como un oikos, como un hogar común y ello ocurre tanto en el Norte como en el Sur, pero más desembozadamente y depredador en el Norte. Recordemos que etimológicamente economía significa el cuidado, el ordenamiento, la administración de la casa, del hogar.

La pretendida integración regional y el desarrollo del comercio a través de acuerdos internacionales, el intercambio de bienes entre naciones, tienen efectos ambientales derivados de la mayor presión sobre los ecosistemas, puesto que estimulan la mayor explotación de los "recursos naturales" y por consiguiente entre otras consecuencias, tanto o más dañinas para el planeta y su gente, aumenta las necesidades de consumo energético y la generación de desechos. Este intercambio en el mercado global de los últimos cincuenta años sigue favoreciendo a los más favorecidos y empobreciendo a los más empobrecidos. La distribución del ingreso per cápita entre los países se ha tornado más desigual en las últimas décadas. En 1960 el PIB per cápita promedio en los 20 países más ricos del mundo superaba en 15 veces aquel de las 20 naciones más pobres. Hoy, esta brecha se ha incrementado hasta 30 veces, puesto que, en promedio, los países ricos han crecido más rápido que los pobres. El ingreso per cápita de los 20 países más pobres se ha mantenido casi inalterado desde 1960, e incluso ha disminuido en varios de ellos ${ }^{21}$.

Por ello los procesos de regionalización y globalización se montan necesariamente sobre un modelo tecnológico, empresarial y de consumo, es decir el modelo económico lucrativo, que sin duda incide de una manera definitiva sobre lo que podría ser un auténtico desarrollo sustentable que tomara en cuenta tanto los factores ambientales como los sociales ${ }^{22}$ : La sociedad capitalista es un sistema de dominio que ha degradado la subsistencia a lo mí- nimo y ha creado la imposibilidad estructural para una reproducción social y ecológica sustentable ${ }^{23}$.

Recordemos la década de los 90 en Argentina cuando el llamado libre juego de los mercados, provocó, así como en toda América Latina un aumento de la pobreza y la inequidad; creció la deuda externa y la degradación ambiental y empeoró la vida tanto en las ciudades como en el campo ${ }^{24}$. Entre 1960 y 1980 la pobreza disminuyó del 51\% al 33,5\% pero, esa cifra volvió a crecer en los 90 y en 2002, "se registraba en la región 221,7 millones de pobres y 98,6 millones de indigentes, la mitad de ellos eran niños o jóvenes ${ }^{24}$. Esto puede ser lamentado incluso en términos capitalistas: ha dado como resultado grandes catástrofes naturales y la explotación desaforada de los recursos, quitándole a muchas sociedades del mundo su capital natural... y poniéndolas en grave desventaja, ya que ese capital se desvaneció para siempre y no puede comprarse en los mercados ${ }^{25}$.

Aunque en principio sólo se veían estas pérdidas en el mundo subdesarrollado, lo ocurrido estos últimos años en los países del Norte, según sucesivos informes del Programa de las Naciones Unidas para el Medio Ambiente (PNUMA), nos obliga a replantearnos una vez más cuál es el riesgo que nos asecha, más allá de detenernos en complacencias regionales circunstanciales. Así como el Norte no podía "salvarse" solo, el Sur tampoco puede hacerlo. Cuando se ve el esfuerzo por "tecnologizarse" de los países latinoamericanos y otros que comparten su condición de explotados por los autodenominados países desarrollados, es necesario tener en claro los riesgos que se asumen, sobre todo por el rol que han jugado la ciencia y la tecnología en la generación o agravamiento de ese riesgo.

Hace mucho tiempo que comenzó la depredación en América Latina y a pesar de las múltiples declaraciones internacionales, sigue su marcha inexorable ${ }^{26}$. Me refiero a América Latina pero la depredación no se limita a ella. Resulta sobrecogedor saber que estamos perdiendo unas 30.000 especies de plantas y animales al año a causa de la perturbación de los ecosistemas y de la caza o recolección excesiva de especies concretas ${ }^{27}$. De modo que no son sólo los técnicos y expertos en recuperación o sustitución de energía o en mantenimiento de la fertilidad del suelo los que van a resolver los problemas ambientales. Plantearlo así sería ver sólo un aspecto de la cuestión y muchas veces estos planteos son tan resonantes e impactantes que ocultan los otros.

En su mayor parte, los problemas ambientales esconden problemas más profundos y ocultos de dominio financiero, desigualdad, pobreza y falta 
de desarrollo equitativo. Ello significa que la solución de estos problemas, como la de los de salud y educación, vitales para el crecimiento de cualquier pueblo, implica que lo que hay que atacar primero es la pobreza y paralelamente los otros factores. Me aventuro a una opinión ya desarrollada en otros textos, donde afirmo que el problema no es la pobreza sino la riqueza. En tanto no consideremos a la riqueza que asociamos con despilfarro, banalidad, exceso de bienes, consumo irrestricto, como el mayor de los problemas la cuestión del uso de los bienes de la tierra no tiene solución.

Implica además darse cuenta que para los países de América Latina, por ejemplo, ocuparse de las cuestiones ambientales es ocuparse de su situación político-económica-social y no de la "naturaleza". Por otra parte, hemos de estar atentos para no caer en la trampa de culpar a los pobres, ni por sus circunstancias ni por sus acciones, porque ellos son las víctimas al igual que la "naturaleza" y no los victimarios.

\section{La sustentabilidad}

La respuesta fácil que aparece frente a estos planteos es que los países subdesarrollados no se desarrollen, ya que eso sería perjudicial para el planeta. Esta respuesta niega la conclusión a que arribamos, puesto que ello significa que los países pobres lo sean aún más a costa de los ricos. De los ricos que tienen nombre: Estados Unidos (EEUU), Alemania, Reino Unido, Francia, Rusia, China, que es donde se asientan los centros de poder monopólico, los centros de inteligencia, el control cultural y científico, el poder armado. La respuesta que buscamos: la sustentabilidad, es global o no es.

El término - sustentabilidad - fue usado por primera vez por la Comisión Mundial del Medio Ambiente y del Desarrollo, constituida por las Naciones Unidas en 1984 para diseñar estrategias que frenaran el deterioro ambiental. Las conclusiones de los estudios de la Comisión se recogieron en el llamado Informe Brundtland, "Nuestro futuro común", en 1988 donde se usó el término y fue definido como el desarrollo que asegura las necesidades del presente sin comprometer la capacidad de las futuras generaciones para enfrentarse a sus propias necesidades ${ }^{28}$.

La sustentabilidad es un proceso que debe comenzar en el Norte que es dónde existe la posibilidad técnica y económica de comenzarlo. ¿Qué podemos hacer desde el Sur? Tener en claro de qué se trata para que no puedan engañarnos con discursos donde se mencionan los derechos de tercera generación. Sobre todo tener en claro que es imposible manteniendo el imperio del sistema capitalista.

Encontramos unidos el discurso sobre la defensa del medio ambiente y el mantenimiento del capitalismo como único sistema económico posible alrededor de concepto de sustentabilidad, cuyo sentido conocemos por aproximación y se usa la mayoría de las veces como un lugar común, vacío de significado. El concepto de sustentabilidad es clásico en economía y aparece asociado actualmente a los problemas ecológicos y del ambiente. La propuesta de lograr un desarrollo sostenible es aceptada por todos los sectores del conocimiento y la política sin discusión, aunque sean pocos los que sepan qué significa cabalmente y menos aún cómo ponerla en práctica.

Como apuntamos antes, hay un supuesto a la base de los que procuran establecer como solución a los problemas ambientales un desarrollo sostenible: asociar desarrollo al crecimiento económico que proviene de la competitividad en los mercados. El desarrollo se presenta siempre como positivo para los países subdesarrollados, cualquiera sea su costo. En el lenguaje económico esos costos incluso tienen un nombre: "externalidades", concepto que hace referencia a costo social, costo ambiental y costo socio-ambiental: Ante el desafío de los costos sociales no incluidos en el precio, las externalidades... se inventan refinanciaciones matemáticas que no logran "internalizarlas" (sobre las cuales poco se sabe, por ejemplo el desmantelamiento de centrales nucleares y sus residuos que duran miles de años) ${ }^{29}$.

El liberalismo económico tiene una respuesta para los que lo acusan de desconocimiento de los problemas ambientales: cuando la renta aumenta, empeora la degradación ambiental hasta un punto, en el cual la calidad medioambiental vuelve a mejo$\operatorname{rar}^{30}$. El discurso desarrollista acepta la degradación, la considera un mal necesario y pasajero en función de un bien mayor. Más allá de que podamos discutir si este costo del desarrollo es justo o deseable, esta relación llamada "de la U invertida" que supone una recuperación del ambiente por sí solo, no considera factores ambientales dispersos y a largo término.

Los factores ambientales que mejoran por sí mismos, incluyen la higiene, la pureza del agua potable, la reducción de partículas de óxido sulfúrico, de óxido de nitrógeno, de monóxido de carbono y de coliformes fecales; no se ha probado que la curva sea válida para el dióxido de carbono, el agotamiento del suelo, la pérdida de bosques y otros procesos basales del ecosistema: La curva de la $U$ invertida 
nos habla de la reducción o recuperación de factores ambientales en sí mismos sin considerar las consecuencias sistémicas. En efecto ignora cuestionen que tienen que ver con lo que sucede a nivel planetario, como por ejemplo, cuando la reducción de un agente contaminante provoca el aumento de otro, o cuando la reducción de la contaminación en un país provoca el aumento de la misma en los países vecinos ${ }^{31}$.

Este tipo de acomodamiento entre las exigencias del ambiente y las propuestas económicas liberales, pretende que sean las prácticas tecnológicas de producción y consumo las que modulen la capacidad de asimilación de la Tierra, desconociendo la complejidad de sus reacciones. Como ejemplo valioso de estas soluciones podemos citar von Weizsäcker y Lovins, donde se busca una fórmula para estimular el reciclaje pero no se hace hincapié en la necesidad de bajar el consumo ${ }^{32}$.

Al asociar esta propuesta al desarrollo, incluso como la única posible como se hace, sólo hay una medida aceptable: el consumo; por consiguiente no sólo es ignorada la dificultad del trato con la "naturaleza" sino el hecho de que sus capacidades son limitadas y que su auténtico potencial no ha podido establecerse. Remedando a Meadows podemos decir que los consumos y emisiones actuales ya son insostenibles y, si se ampliaran a los países del Sur los actuales niveles de consumo de los países del Norte, la situación ecológica del mundo sería mucho peor, ya que se requerirían 10 veces más recursos ${ }^{33}$. Como afirma Nicholas Georgescu-Roegen en su propuesta de economía ecológica, ${ }^{34}$ la naturaleza debe ser asumida como límite para los procesos económicos

No está de más recordar estos datos: entre 1950 y 2007 el consumo de agua se ha triplicado, el de combustibles fósiles se ha quintuplicado, el de carne creció un $550 \%$, las emisiones de dióxido de carbono han aumentado un $400 \%$, el PIB mundial aumentó un $716 \%$, el comercio mundial llegó a un $1.568 \%$, el gasto mundial en publicidad creció un $965 \%$, el número de turistas que salieron de sus fronteras aumentó un $2.860 \%$, el número de automóviles pasó de 53 millones en 1950 a 565 millones en 2002 y el consumo de papel saltó a un $423 \%$, en este caso entre 1961 y $2002{ }^{33}$. Por ello, pensar el desarrollo según las pautas actuales es una trampa mortal. No es ilógico, entonces buscar como solución mantener el subdesarrollo donde éste ya existe.

La vida humana conforma un espacio significativo, un hábitat, un ambiente que lo va conformando, el aire que respira y lo que come lo hace ser quién es y dar sentido a su vida, de la misma manera que los condicionamientos sociales y el uso del len- guaje, por lo cual debe reconocer que la fertilidad de la tierra, el agua y el aire forman parte de sus referencias simbólicas y que ignorarlo constituye una "especie" de ser humano empobrecido y sin horizonte vital. Ya los griegos habían notado que el hombre como todo ser es un compuesto de aire, tierra y agua. ¿Es posible entonces vivir dignamente, donde el agua, el aire y la tierra se conviertan en elementos hostiles, enemigos, venenos contaminantes? ¿O instrumentos que uso y tiro?

Cualquier respuesta ética o política, cualquier arquitectura social, se establece solidariamente con la concurrencia ignorada de estos elementos. Ningún proyecto humano es factible sin tierra, agua y aire. Pensar que no habrá degradación ambiental si los países subdesarrollados no se desarrollan es no sólo injusto sino insensato.

\section{Consideraciones finales}

Mientras se sigan planteando los problemas específicamente, como cuestiones separadas, el ambiente será un problema de expertos. Mientras no se tome una actitud de exigencia ética frente al desarrollo aplastante de la tecnología y biotecnología todo discurso será de escasa trascendencia. Si bien el factor desencadenante de este estado de riesgo ambiental no es la tecnología, sino la codicia y la ambición humana sin límite, uno de los primeros pasos a dar es dejar de considerar a la tecnología como un destino inexorable y tomar distancia de ella, una distancia asumida vitalmente y no como mera retórica. El manoseado valor de la coherencia debe ser recuperado y aplicado al modo que tenemos de enfrentar los problemas. Vimos que lo que exige un desarrollo sostenible son decisiones políticas que hagan planes considerando la interrelación de tres elementos básicos: justicia económica, sostenibilidad y protección de los ecosistemas e igualdad social.

Cuando hablamos de desarrollo sostenible lo estamos tomando como sinónimo de desarrollo, perfeccionamiento, florecimiento lo cual implica acciones diferentes que si lo asociamos a: aumento, crecimiento, incremento, opulencia. En el primer sentido no sólo no agotaría los recursos de la "naturaleza" sino que les permitiría acompañar como lo hace desde que el hombre es hombre, el acontecimiento del bios: la manifestación de las diferentes culturas. Se trataría de una sustentabilidad apoyada sobre la justicia, el reconocimiento del valor del otro, sea humano o no. Para ello habrá que remon- 
tar una prédica escéptica frente a la ética, que la suplanta por la eficacia.

En la época en que las ideologías desaparecieron, este pragmatismo actúa en su lugar y adquiere incluso carácter ideológico, se cuela por la ventana enmascarado detrás de grandes palabras de peso moral tradicional que no son puestas en duda ni a las que se les pregunta qué significan y cuyo resultado es el dominio no sólo de la "naturaleza" sino del humano. Palabras como autonomía, equidad, libertad, derecho, progreso, ciencia, bioética, se escriben con mayúscula, y la que se escribe con una mayúscula aún mayor es justicia aunque lo que se entiende por ella es poder ir a un tribunal presidido por un juez y pleitear al amparo de una ley. No cuestiono aquí ni el pleito, ni la ley ni al juez, solo que justicia y con mayúscula hace referencia a algo que trasciende la ley. Este mismo tipo de falacia se repite con las otras grandes palabras como solidaridad. Cuando el pragmatismo liberal exige que se actúe éticamente está reclamando que intervenga un juez que le proponga a una persona elegir libremente ejerciendo su autonomía y no considerándola inferior a nadie, cualquiera sea su estado, entre opciones manifiestas.

Sin embargo, esto que es necesario, no basta sino que se convierte en una caricatura de una genuina ética que busca la justicia. Muchas veces este acto puede ser la resultante de un auténtico ejercicio de la justicia pero en la mayoría de los casos se convierte en un simulacro en que todos actúan como si las personas fueran libres, iguales y obraran como sujetos de derecho, pero no es así en sociedades fundamentalmente condicionadas económicamente, socialmente, históricamente. Para el ejercicio de la ética, por consiguiente, no basta una decisión o una elección en soledad sino que ésta debe implicar la asunción de una responsabilidad social. La responsabilidad es "el deber del poder" 35 , y en consecuencia ocupa un lugar nuclear en el ámbito de la ética cuando ésta deriva en acción política, es decir acción pública solidaria y comprometida con el otro humano o no humano. La responsabilidad implica la solidaridad ${ }^{36}$.

Nada de lo que hacemos termina en cada uno de nosotros sino que se "extiende" en el espacio, tiene consecuencias sobre los otros y viceversa. No podemos pensar ninguna relación sana, no es posible concebir la salud sin los otros entre los que debemos contar el mundo "natural" ${ }^{37}$. Los hombres somos solidarios de nuestra suerte común y de la que sufra nuestro planeta. O nos salvamos juntos o pereceremos juntos. Ningún movimiento en este mundo que vivimos es en vano, ni en el mundo denominado na- tural ni en el cultural, todo movimiento es solidario de por sí, nos sumerge en un movimiento total. Es el ejercicio libre de la corporalidad como habitación del espacio común lo que permite al humano reconocerse arte y parte del "mundo natural" ${ }^{38}$.

Solidaridad es entonces la primer respuesta, la que nos permitirá vivir de la mano con el prójimo y no compitiendo con él, la que nos dará la chance de construir en común en vez de destruirnos unos a otros, la que nos permitirá reconocer la vida a nuestro alrededor, la que permitirá una auténtica sustentabilidad. Y la solidaridad exige el compromiso, es decir la promesa común, el prometernos, entregarnos los unos a los otros, confiando unos en otros.

La ética no puede imponerse, no tiene con qué hacerlo, sólo es una formulación de criterios de acción que sólo valen si son asumidos como comportamientos. La eficacia de la ética sólo puede medirse cuando su tensión interna que se manifiesta en una vocación de libertad, de justicia, se transforma en decisiones y acciones políticas que responden a esa tensión. En ese marco, el reclamo es que las prácticas científicas y tecnológicas, toda intervención sobre la vida humana y de la "naturaleza" se realicen bajo las exigencias de la ética. Ésta debe ser el sustento de toda acción política que incluye la de los estados, en los que también debe manifestarse esa tensión. Si bien el ejercicio de la ética no puede ser separado del de la política, acentuar el primero nos aparta de la tentación de un pragmatismo utilitario y nos permite subrayar lo que tenga que ver con los valores que enervan las acciones humanas comunitarias, como son el valor de la vida humana, de la identidad, de la integridad, de la libertad. Acompañar la acción política con la reflexión ética nos obliga a respetar la vida y más aún a honrarla, es decir enaltecerla.

La réplica de la ética frente a los cambios generados por la mano del hombre en la "naturaleza", que se nos presentan como riesgos, incluso como peligros y amenazas para nuestro futuro, debe ser en primer lugar reclamar a la tecnociencia que los provoca, una respuesta comprometida y referir esa respuesta al principio de precaución ${ }^{39,40}$. Las consideraciones técnicas, las propuestas ecológicas, las reflexiones filosóficas respecto de la relación humano-"naturaleza" deben caer en tierra fértil y ello sólo es posible cuando caigan en tierra de justicia. Pero la justicia no debe ser abstracta sino que debe verse puesta en acto en la política de los estados. Ello sólo será posible desde la reversión de algunas situaciones que son injustas en sí mismas como por ejemplo las diferencias actuales en el uso de recursos naturales entre el Norte y el Sur en favor del Norte, la 
subsidiariedad de la economía del Norte por la del Sur siendo que este es pobre y aquél rico, el carácter depredador de la economía, no sólo de recursos "naturales" sino humanos.

La Declaración de Río en 1992 decía: El mejor modo de tratar las cuestiones ambientales es mediante la participación de todos los ciudadanos interesados en el nivel que corresponda. En el plan nacional, cada individuo deberá tener acceso adecuado a la información relativa al medio ambiente de que disponen las autoridades públicas, incluida la información sobre los materiales y actividades que ofrecen peligro en sus comunidades, así como la oportunidad de participar en los procesos de adopción de decisiones. Los Estados deberán facilitar y fomentar la sensibilización y la participación del público poniendo la información a disposición de todos. Deberá proporcionarse acceso efectivo a los procedimientos judiciales y administrativos, entre éstos, el resarcimiento de daños ${ }^{41}$. Por lo tanto, lo primero a poner en la mira es lograr sociedades en que los recursos, sean pocos o muchos, estén al alcance de todos los ciudadanos por igual y en que la acción política no sea sólo cosa de los gobiernos pero sea responsabilidad de los estados.

\section{Referências}

1. Amorín C. Ciencia, conciencia y sociedad. Mae-Wan Ho Uruguay. Biodiversidad. 2000 out; 25-26: 33. Disponível: http://www.grain.org/es/article/entries/907-ciencia-conciencia-y-sociedad-dramae-wan-ho-en-uruguay (acesso 10 nov. 2011).

2. MerinoS. Latragedia deSanta Fe. RedLatinoamericanay del Caribe de Bioética. [Internet] Disponível: http://www.unesco.org.uy/shs/red-bioetica/es/noticias-y-opiniones/bioetica-y-sociedad. html (acesso 18 jun. 2014).

3. Marraro F. Siembra soja y cosecharás inundados. Fundación Proteger. 23 maio 2004. Disponível: http://www.proteger.org.ar/siembra-soja-cosecharas-inundados (acesso 10 nov. 2011).

4. Samsel A, Seneff S. Supresión de enzimas citocromo P450 y biosíntesis de aminoácidos del microbioma intestinal por glifosato: via de enfermedades modernas. Entropy. 2013; 15:4: 1.41663. Disponível: www.mdpi.com/1099-4300/15/4/1416 (acesso 18 jun. 2014).

5. Avila V, Medardo. Sr. Ministro Barañao el glifosato no es agua con sal. [Internet] Red Universitaria de Ambiente y Salud, Médicos de pueblos fumigados. Disponível: http://www.lavoz.com.ar/ opinion/ministro-baranao-glifosato-\%EF\%BF\%BDno-es-agua-con-sal (acesso 10 nov. 2011).

6. Red Universitaria de Ambiente y Salud. Lo que no se dice de la nueva planta de Monsanto en Córdoba. [Internet] 5 set. 2012. Disponível: http://www.reduas.fcm.unc.edu.ar/lo-que-no-sedice-de-la-nueva-planta-de-monsanto-en-cordoba (acesso 18 jun. 2014).

7. Greenlee AR, Arbuckle T, Chyou P. Risk factors for female infertility in an agricultural region. Epidemiology. 2003 jul.; 14(4): 429-36.

8. Der Parsehian S, Grandi D. Plaguicidas organoclorados prohibidos en leche materna. Actas $33^{\text {er }}$ Congreso Argentino de Pediatría. Mar del Plata; 1 al 4 de Octubre del 2003.

9. Weber M. El político y el científico. Madrid: Alianza Editorial; 1967.

10. Apel KO. Diskurs und Verantwortung. Das problem des Übergangs zur postkonventionellen moral. Frankfurt: Suhrkamp; 1988.

11. Farvar MM, John P, editors. The careless techology. Ecology and international development. Garden City: The Natural History Press; 1992. p. 960.

12. Pengue W. Fundamentos de economía ecológica. Buenos Aires: Kaicron; 2009.

13. Martínez Alier J, editor. Los principios de la economía ecológica. Madrid: Argentaria; 1995.

14. El Informador. La ONU alerta que el planeta está "en peligro". Disponível: http://www.informador. com.mx/internacional/2013/452738/6/la-onu-alerta-que-el-planeta-esta-en-peligro.htm

15. Heidegger M. Bauen, Vohnen, Denken. In: Heidegger. Vortrâge und Aufsätze. Pfullingen: Neske; 1954.

16. Pfeiffer ML. "El mal radical”. Agora. Papeles de Filosofía. 2000 fev.; 19(2): 127-38.

17. Flores M. Todos los caminos conducen a la propiedad intelectual. Revista Biodiversidad. 2002 jan; 31:8.

18. Meadows DH, Randers J, Behrens W. Los límites del crecimiento. Madrid: Fondo de Cultura Económica; 2002.

19. Meadows DH, Randers J, Behrens W. Los límites del crecimiento 30 años después. Galaxia Gutenberg: Barcelona; 2006.

20. Latouche S. La apuesta por el decrecimiento: ¿cómo salir del imaginario dominante? Barcelona: Ed. Icaria; 2009.

21. Stiglitz J. El malestar en la globalización. Buenos Aires: Taurus; 2002.

22. Buxedas M. El desarrollo sustentable en las negociaciones del Mercosur. Nueva Sociedad Caracas. 1999 jul-ago; 162: 68-78.

23. Breilh J. Perspectivas política, sociales y éticas de la investigación en una era de barbarie. Rev. Esc. Enferm. USP. 2002; 36(3):214. 
24. Rodriguez M, Calvento M. Análisis de los efectos de las crisis internacionales sobre la pobreza en América Latina: El caso de Argentina. Observatorio de la Economía Latinoamericana. 2004 out; 33. Disponível: http://www.eumed.net/cursecon/ecolat/ar/ (acesso 10 nov. 2011).

25. Reboratti C. Ambiente y sociedad. Buenos Aires: Planeta/Ariel; 1999. p. 216.

26. Pfeiffer ML. Transgénicos. Un destino tecnológico para América Latina. Mar del Plata: Ed. Suárez; 2002.

27. Eldrege N. La vida en la cuerda floja. La humanidad y la crisis de la biodiversidad. Barcelona: Tusquetes; 2001.

28. Organización de las Naciones Unidas. Informe Brundtland "Nuestro futuro común". (ONU/ Comisión Mundial para el Medio Ambiente y el Desarrollo; 1988) [Internet] Disponível: http:// daccess-dds-ny.un.org/doc/UNDOC/GEN/N87/184/67/IMG/N8718467.pdf?OpenElement. (acesso 10 nov. 2011).

29. Ramos Martín J. Empirismo en economía ecológica: una visión desde la teoría de los sistemas complejos. 2004. [Internet] Disponível: http//www.geocities.com (acesso 30 out. 2011).

30. Kenneth Arrow, Bolin B, Costanza R, Dasgupta P, Folke C, Holling CS et al. Economics growth carrying capacity and the environment. Science. 1995 abr.; 268: 520-1.

31. Carpenter S. Desarrollo y sostenibilidad fuerte. AAVV ¿Sostenible? Barcelona: Ed. Icaria; 1997. p. 58-9.

32. von Weizsäcker EU, Lovins AB, Lovins LH. Factor 4. Duplicar el bienestar con la mitad de recursos naturales. Informe al Club de Roma. Barcelona: Galaxia Gutenberg/Círculo de Lectores; 1997.

33. Pengue W. Revolución de la sostenibilidad. Entrevista. 2011 [Internet] Disponível: http://vallianceprod.com/index.php?option=com_content\&view=article\&id=68\&ltemid=63\&lang=es (acesso 10 nov. 2011).

34. Georgescu-Roegen N. La ley de la entropía y el proceso económico. Fundación Argentaria, Madrid, 1999.

35. Jonas H. El principio de responsabilidad. Madrid: Herder; 1995.

36. Pfeifer ML. Todos para uno y uno para todos. El principio de solidaridad. In: D. Michelini. Libertad, solidaridad, liberación. Homenaje a J.C. Scannone. Río Cuarto: Ediciones del Icala; 2003.

37. Junges JR, Barbiani R. Interfaces entre território, ambiente e saúde na atenção primária: uma leitura bioética. Rev. Bioét. (Impr.). 2013: 21(2): 207-17.

38. Pfeiffer ML. El espacio de la desmesura. Una aproximación bioética a la moral. Buenos Aires: Antropofagia; 2014. (en prensa).

39. Pfeiffer ML. El principio de precaución. In: G. Fernández, S. Cecchetto, editores. Transgénicos en América Latina: el retorno de Hernán Cortés. Mar del Plata: Suárez; 2003.

40. Agencia Europea de Medio Ambiente. Lecciones tardías a partir de alertas tempranas: el principio de precaución 1896-2000. Madrid: Centro de Publicaciones del MMA; 2003.

41. Organización de las Naciones Unidas. Declaración de Río sobre el medio ambiente y el desarrollo. (ONU;1992) Principio 10 [Internet] Disponível: http://www.pnuma.org/docamb/dr1992.php

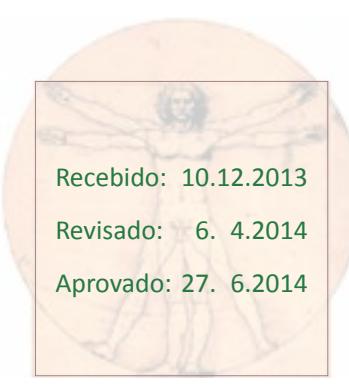

